\title{
The ocular and systemic prognosis of patients presenting with sarcoid uveitis
}

\author{
Abstract \\ Purpose To describe the visual and systemic \\ outcomes in patients presenting with sarcoid \\ uveitis.
}

Methods Seventy-five patients with definite or presumed sarcoid uveitis were followed up for a median of 4 years. The patients came from a primary ophthalmic referral centre and a specialist uveitis centre. The prognostic value of demographic and clinical features at the onset of disease were studied. Baseline and outcome variables were analysed by survival analysis.

Results After 10 years, 54\% of patients retained normal visual acuity and $4.6 \%$ had lost vision to less than $6 / 36$ in both eyes. Fiftyone per cent required oral steroids for uveitis and a further $11 \%$ needed additional immunosuppressants. Twenty-one per cent of patients had undergone a surgical procedure. At the onset of uveitis the lung was the most common organ involved (35\%). After 10 years follow-up disease spread to other organs in 13 patients $(17 \%)$; in 8 of 13 patients this was the central nervous system. The only outcome associated with baseline variables was bilateral visual loss, which was more likely in those over 40 years at presentation $(p=0.004)$. Conclusions The ocular prognosis of sarcoid

C. Edelsten

E. Joynes

M.R. Stanford

E.M. Graham

The Medical Eye Unit St Thomas' Hospital Lambeth Palace Road London, UK

\section{Edelsten}

A. Pearson

The Prince Charles Eye Unit King Edward VII Hospital Windsor, Berkshire, UK

Mr Clive Edelsten Ipswich Hospital NHS Trust Heath Road

Ipswich IP4 5PD, UK

Tel: +44 (0)1473 712233

Received: 7 May 1998 Accepted in revised form: 27 July 1999 uveitis is unrelated to the extent of disease at onset. Patients with extraocular disease fared no differently from those with isolated ocular disease. Patients with sarcoid uveitis are at risk of neurological involvement for at least 15 years.

Key words Epidemiology, Sarcoidosis, Uveitis, Visual outcome

Patients with pulmonary sarcoidosis may develop uveitis and patients presenting with idiopathic uveitis may later develop extraocular sarcoidosis. The benefit of screening uveitis patients for occult sarcoidosis in asymptomatic sites has not been systematically studied. Chest radiography and the measurement of serum angiotensin converting enzyme (sACE) are the most commonly performed tests in the United Kingdom, but there are great variations in the
C. EDELSTEN ${ }^{1,2}, A \cdot$ PEARSON ${ }^{2}$,

E. JOYNES ${ }^{1}$, M.R. STANFORD ${ }^{1}$,

E.M. GRAHAM ${ }^{1}$ extent to which these and other diagnostic tests are performed on patients with chronic uveitis. The benefits of more invasive tests for sarcoidosis, such as tissue biopsy and isotope scanning, must be set against their cost and danger to patients. There remains no cure for sarcoidosis and treatment for extraocular disease is usually restricted to those patients with relevant symptoms. Knowledge of the prevalence and likely site of symptomatic sarcoidosis in patients with uveitis is therefore needed to guide the appropriate use of invasive testing in these patients.

Sarcoid uveitis is a major cause of visual loss in North European patients with uveitis ${ }^{1}$ and early identification of those patients with a poor prognosis may help to reduce their long-term visual loss. The majority of studies on the outcome of sarcoid uveitis have examined patients known to have pulmonary sarcoidosis. ${ }^{2-4}$ There are many patients with uveitis that is clinically identical to that associated with biopsy-proven pulmonary sarcoidosis but in whom a definitive diagnosis has not been established. Sarcoidosis is commonly defined as a multi-system disorder and disease confined to the eye should strictly, therefore, be excluded from this definition. It is not currently known whether the uveitis associated with pulmonary sarcoidosis has a similar course to the uveitis associated with other forms of the disease.

We therefore wished to determine whether the distinction between patients with definite pulmonary sarcoidosis and other less welldefined forms of the disease is clinically relevant to the long-term management and ocular prognosis of patients with uveitis. In this study we examined the long-term course of patients with confirmed and presumed sarcoid uveitis and evaluated the risk factors associated with a poor prognosis. In particular, we examined whether the prognosis for patients with no clinical evidence of extraocular sarcoid at the onset of their uveitis was any different from those with symptomatic pulmonary disease, and also determined which organ systems were most likely to develop disease after the onset of uveitis. 


\section{Materials and methods}

All patients with uveitis and evidence of sarcoidosis who attended the Medical Eye Unit, St Thomas' Hospital, London, and The Prince Charles Eye Unit, Windsor, from 1991 to 1994 were included in the study. The former is a tertiary referral centre for inflammatory eye disease and the latter is a general district ophthalmic unit. The time from the onset of uveitis to the last outpatient visit varied from 1 to 48 years. There was considerable variation in the nature and timing of the investigations performed to confirm the diagnosis as these depended on both the nature of the clinical presentation and the year of onset. The minimum evidence of sarcoidosis required for inclusion into the study was any of the following: an otherwise unexplained raised sACE level, bilateral hilar lymphadenopathy on chest radiograph or a positive Kveim test. All patients had chest radiographs and sACE measurement.

The patients consisted of three groups. The first was those with clinical and radiological evidence of pulmonary sarcoidosis within the first year of the onset of uveitis. The secondary included those with clinical or biopsy evidence of extraocular sarcoidosis within 1 year of the onset of uveitis but no clinical or radiological evidence of pulmonary disease. The third group comprised those patients with uveitis compatible with sarcoid uveitis, and no clinical or radiological evidence of extraocular sarcoidosis at the onset of uveitis, who had an otherwise unexplained rise in SACE and/or a positive Kveim test.

\section{Methods and statistical analysis}

Demographic and clinical variables at the onset of uveitis were correlated with several outcome measures. The baseline variables included sex, race and age as well as the type of organ involvement at the onset of uveitis (as defined above). Outcome variables included visual acuity, raised intraocular pressure requiring treatment, any intraocular surgery, new symptomatic sarcoidosis in an organ uninvolved at baseline, the use of systemic steroids to control intraocular inflammation and the use of an immunosuppressant in addition to, or instead of, oral steroids.

Permanent changes in visual acuity were grouped into four exclusive, ranked levels: 1 (mild unilateral), $6 / 12-6 / 24$ in one eye only; 2 (severe unilateral), $6 / 36$ or worse in one eye only; 3 (mild bilateral), 6/12-6/24 in both eyes; and 4 (severe bilateral), $6 / 36$ or worse in both eyes. Normal acuity was defined as better than $6 / 12$ in each eye. Temporary changes in acuity were excluded. A drop in acuity was defined as permanent if it had not improved with 1 year of follow-up and reversible causes such as treatable macular oedema or cataract had been excluded. Systemic steroids were used conventionally for the treatment of posterior uveitis, i.e. for control of persistent posterior segment inflammation reducing the visual acuity to $6 / 12$ or less. Other immunosuppressants were used as steroid-sparing agents or, rarely, to replace steroids when they produced unacceptable side-effects. Additional immunosuppressants were used neither routinely with steroids nor as primary monotherapy. In all analyses individual patients were used as cases and not individual eyes. The outcome of uveitis was rarely symmetrical and we therefore included categories of visual loss with both symmetrical and asymmetrical visual loss in order to maximise the power of the analysis. Initial analysis compared the demographic and clinical variables between the two hospitals. Further analysis aggregated the two populations. The main outcome measure of visual acuity was correlated with the baseline variables and the other outcome measures using chi-squared tests. The relationship between baseline variables and the various outcome measures was then analysed by Kaplan-Meier statistics.

\section{Results}

\section{Demographic and baseline characteristics}

Seventy-five patients were studied. There were 41 females (55\%). Sixty were white and 15 (20\%) were not, comprising 10 Asians, 4 Afro-Caribbeans and one Oriental. The median age at the onset of uveitis was 36 years (range 10-86 years) and 41 (55\%) were over 40 years old. The median follow-up time was 4 years (range 1-48 years): 37 were followed for 1-3 years, 15 were followed for 4-6 years, 11 were followed for 7-12 years and 12 were followed for $13-48$ years. Thirty-five (47\%) patients had extraocular sarcoid at the onset of uveitis; these included 24 (35\%) with pulmonary sarcoid and 11 $(12 \%)$ with extrapulmonary disease only. Sixteen patients had more than one organ system involved (Table 1).

Thirty-eight patients required both systemic and topical treatment. Five had systemic treatment only, 33 had topical treatment only and 4 had had no treatment for uveitis by the time of their last follow-up visit. The median time that patients were given topical treatment was 2 years (range 1-29 years); the median time on systemic treatment was also 2 years (range 1-10 years). Fifty-one patients were from St Thomas' Hospital and 24 from Windsor. They were similar in age, sex and race but patients from St Thomas' Hospital were more likely to have extraocular sarcoidosis at the onset of the uveitis $(31 / 51,60 \%)$ compared with patients from Windsor $(4 / 24,17 \% ; p=0.003)$. Patients at St Thomas' Hospital

Table 1. Pattern of extraocular involvement at the onset of uveitis in 35 patients

\begin{tabular}{lr}
\hline Lung & 24 \\
Lymph node & 8 \\
Skin & 7 \\
Joint & 7 \\
Central nervous system & 4 \\
Liver & 3 \\
Epididymis & 2 \\
Kidney & 1 \\
Muscle (calf) & 1 \\
Nose & 1 \\
Heart & 1 \\
\hline
\end{tabular}




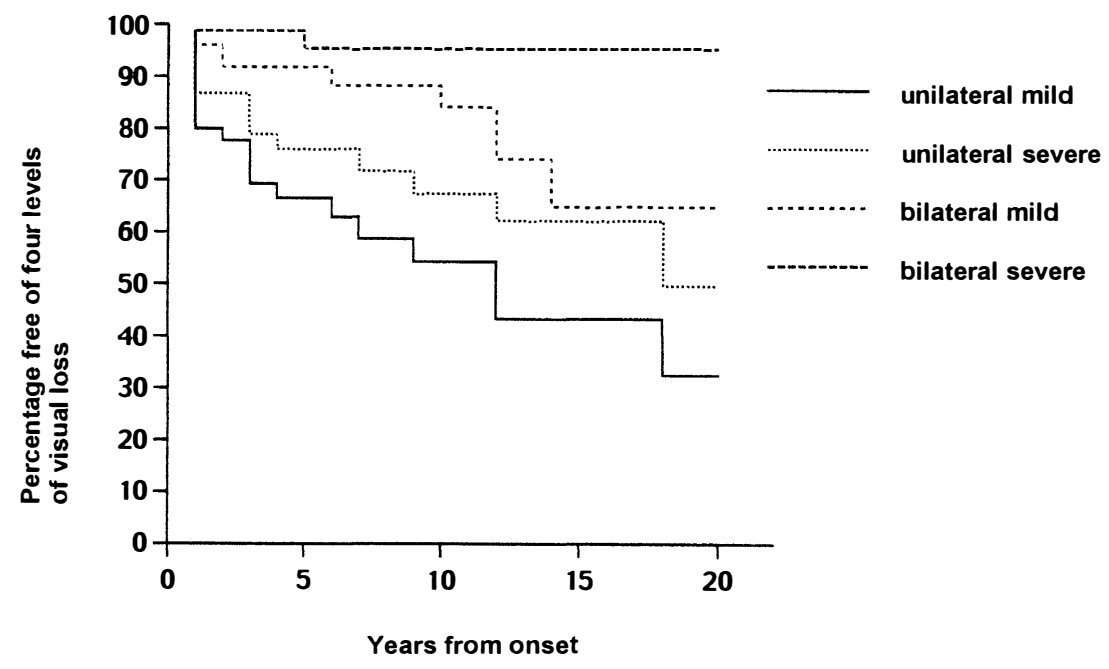

Fig. 1. Kaplan-Meier analysis of visual outcome in sarcoid uveitis. Permanent reduction in visual acuity to four categories of visual impairment: mild unilateral, severe unilateral, mild bilateral and severe bilateral (see text for definitions).

included tertiary referrals for complications of systemic sarcoidosis and therefore have a greater frequency of patients with extraocular disease.

\section{Analysis of outcome}

The last recorded visual acuity was reduced to $6 / 36$ or less in both eyes in 3 patients $(4 \%), 6 / 12$ or less in both eyes in $7(9 \%), 6 / 36$ or less in one eye only in 9 patients $(12 \%)$, and $6 / 12$ or less, in one eye only, in $8(11 \%)$. After 10 years, $54 \%$ of patients retained normal acuity and $4.6 \%$ had severe bilateral visual loss (Fig. 1).

Thirty-eight (51\%) patients required oral steroids; 8 (11\%) also required a second immunosuppressive agent. Five patients had azathioprine, one had cyclophosphamide, one had cyclosporin, and one had both azathioprine and cyclosporin A.

Sixteen patients $(21 \%)$ required ocular surgery. The procedures included 13 cataract extractions, three trabeculectomies, one retinal detachment and one vitrectomy and membrane peel. The mean time after the onset of uveitis for cataract extraction was 8.2 years (range 1-28 years). Ten patients (13\%) developed significantly raised intraocular pressure requiring medical treatment, but only 3 required trabeculectomy.

Symptomatic spread of sarcoidosis occurred in 13 patients. The organ systems involved included the central nervous system in 8 patients ( 3 optic neuropathy, 3 cord lesions, 1 hemiplegia, 1 cranial nerve palsy), the skin in 2 patients, and individual patiens with lung, joint, spleen and lymph node involvement. Neurological involvement occurred a mean of 8 years (range 1-16 years) after the onset of uveitis. Optic neuropathy included episodes of presumed sarcoid neuropathy distinct from papillitis or persistent disc oedema associated with posterior uveitis.

There were no significant differences in the levels of steroid use, visual acuity, surgery or disease spread between the two patient populations. All patients who had additional immunosuppression were seen in St Thomas' Hospital $(8 / 51$ vs $0.24 ; p=0.04)$. There were more patients with raised intraocular pressure at Windsor $(6 / 24$ vs $4 / 51 ; p=0.04)$. There were no obvious differences in the indications for treatment between the two hospitals. Differences in immunosuppression and glaucoma rates are probably due to differences in referral patterns. Patients are referred to a tertiary referral centre when steroid treatment is inadequate or not tolerated: secondary glaucoma is not a common reason for referral and the relative proportion of patients with glaucoma will be higher in district ophthalmic centres.

Patients with reduced visual acuity compared with those with normal visual acuity at the end of the study were more likely to have had ocular surgery, raised intraocular pressure and to have used oral steroids (Table 2). These parameters were most strongly associated with mild unilateral loss of vision and there was no significant association with the two categories of bilateral visual loss. The main reason for permanently reduced vision was irreversible maculopathy arising from posterior segment inflammation requiring steroids or glaucomatous damage and thus these correlations between outcome variables were to be expected. There

Table 2. The frequency of ocular hypertension, ocular surgery and the use of oral steroids in those with normal acuity and those with at least mild unilateral visual loss

\begin{tabular}{llcc}
\hline & Raised IOP & Surgery & Oral steroids \\
\hline Acuity $>6 / 12$ both eyes & $3 / 48(6.3)$ & $5 / 48(10.4)$ & $18 / 48(37.5)$ \\
Acuity $\leqslant 6 / 12$ in at least one eye & $8 / 27(29.6)$ & $11 / 27(40.7)$ & $20 / 27(74.0)$ \\
\hline
\end{tabular}

Percentages are shown in parentheses. All outcomes are significantly increased in those with visual loss (all $p=0.002$ ). 


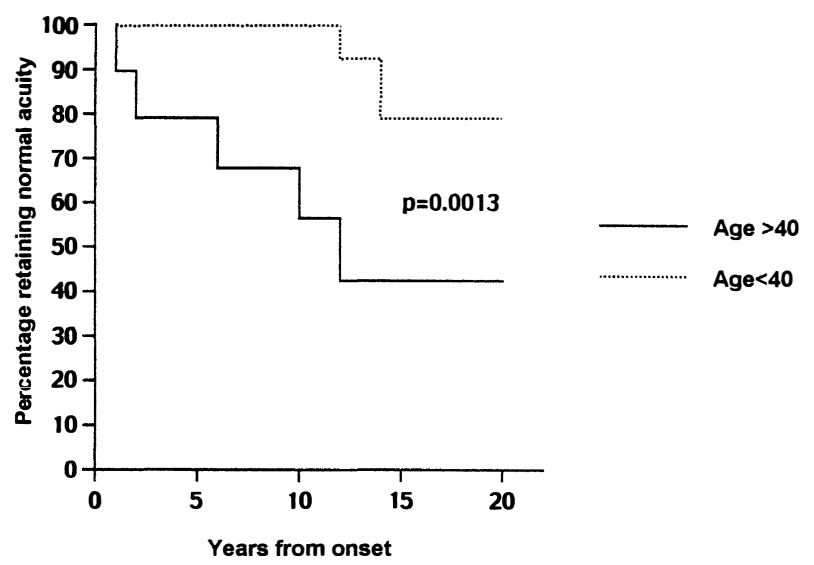

Fig. 2. Difference in rate of mild bilateral visual loss in sarcoid uveitis by age of onset. Patients 40 years or older at the onset of uveitis lose vision at a significantly greater rate for at least 10 years.

was no association between final visual acuity and the use of second-line immunosuppression or spread of disease.

\section{Relation between baseline variables and outcome}

Bilateral visual loss was associated with later onset of uveitis. In patients under the age of 40 years, 2 of $46(4 \%)$ lost vision to less than $6 / 12$ in both eyes compared with 8 of $29(28 \%)$ of those whose uveitis started after this age $(p=0.004)$. There was no significant association of age with the two categories of unilateral visual loss. Spread of sarcoidosis was marginally more common in younger patients, with 11 of 35 (31\%) under 40 years developing new disease compared with 2 of $27(7 \%)$ older patients $(p=0.06)$. There was no relation between any outcome variables with sex, race or the pattern of organ involvement at the onset of uveitis.

Mean visual acuity declined steadily over the followup period (Fig. 1). The rate of irreversible visual loss was similar for the first three levels of visual acuity, with $2.8 \%$ of patients suffering further visual loss each year. Severe bilateral visual loss was rare and only developed in the

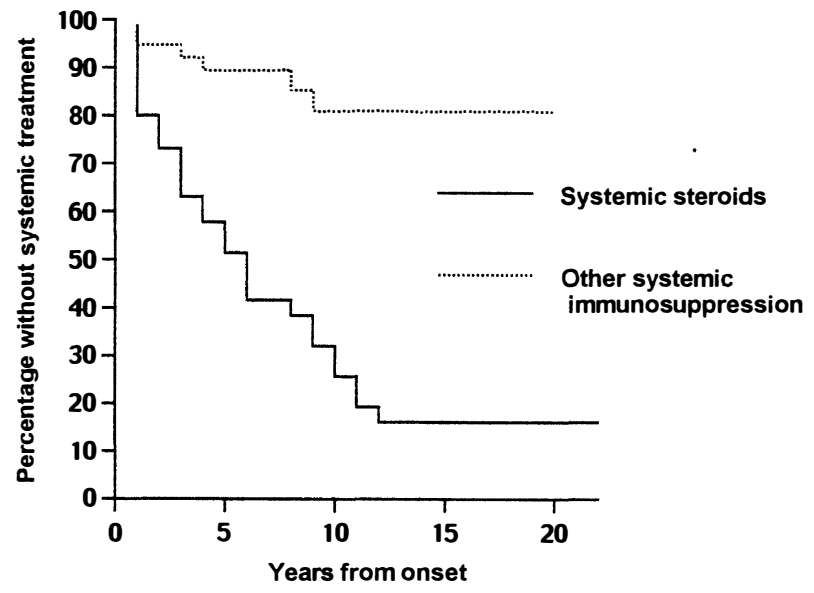

Fig. 3. Rate of use of oral steroids or additional oral immunosuppression in sarcoid uveitis (Kaplan-Meier analysis).

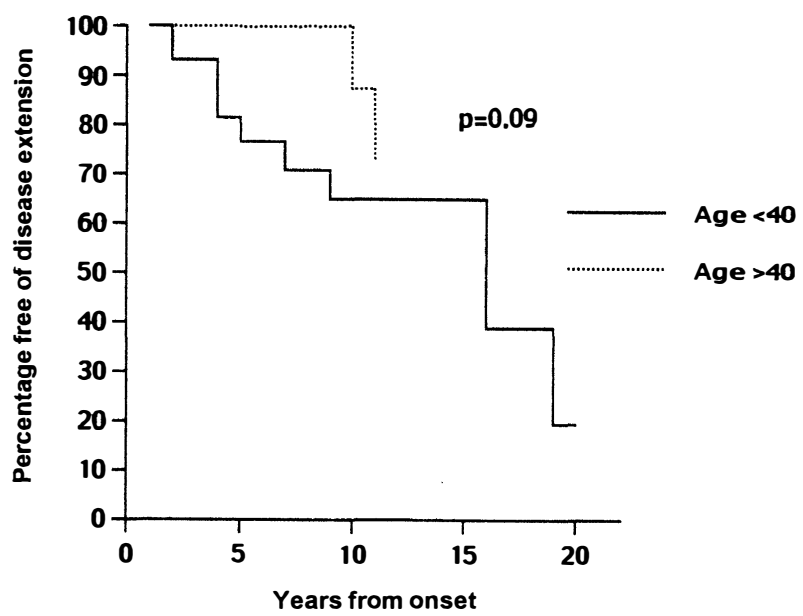

Fig. 4. Rate of extraocular sarcoidosis 1 year after the onset of uveitis (Kaplan-Meier analysis).

first 5 years. The rate of lesser levels of visual loss did not appear to decrease with time. The rate of mild bilateral visual loss was significantly greater in those whose uveitis began after 40 years of age $(p=0.001)$. After 15 years, $60 \%$ of older patients had lost acuity compared with $20 \%$ of younger patients (Fig. 2).

Fifteen per cent of patients required surgery after 5 years and 39\% after 15 years; $8 \%$ developed ocular hypertension after 5 years and $19 \%$ after 15 years. There did not appear to be any diminution of risk over time (Table 3).

Oral steroids were initiated in $49 \%$ after 5 years and $84 \%$ after 15 years. Additional immunosuppression was required in $10 \%$ at 5 years and $19 \%$ at 15 years (Fig. 3). The risk of requiring systemic steroids appeared to decline after 12 years, and the risk of requiring additional immunosuppression appeared to decline after 9 years. Further spread of sarcoidosis occurred in 15\% after 5 years and $32 \%$ after 15 years. There was a trend for younger patients to be more likely to develop spread of disease (Fig. $4 ; p=0.09$ ). There was no significant correlation of the baseline variables with the risk of ocular surgery, ocular hypertension, oral steroids or additional immunosuppression.

\section{Discussion}

Sarcoidosis is difficult to diagnose and the majority of those with evidence of disease at post-mortem have not been previously diagnosed. ${ }^{5,6}$ Any study of sarcoid uveitis is hampered by the impossibility of achieving a certain diagnosis, particularly where ocular disease is present on its own, where there is a lack of universally

Table 3. Prevalence of complications 5 and 10 years after the onset of uveitis: Kaplan-Meier probability

\begin{tabular}{lcc}
\hline Complication & 5 years (SE) & 10 years (SE) \\
\hline Oral steroids & $48.6(7.7)$ & $74.3(7.5)$ \\
Other immunosuppression & $10.4(4.3)$ & $18.9(6.9)$ \\
Ocular hypertension & $7.5(3.8)$ & $19.3(7.2)$ \\
Ocular surgery & $14.9(5.5)$ & $26.3(7.8)$ \\
Spread of sarcoidosis & $14.9(5.7)$ & $27.1(8.1)$ \\
\hline
\end{tabular}


recognised diagnostic criteria and where wide variations in the sensitivity of the diagnostic tests available exist. It was not our purpose to investigate the efficacy of our diagnostic methods, but to describe the clinical problems that arise in these patients and thereby clarify the role of invasive diagnostic tests.

The prevalence of sarcoidosis in large European series of uveitis patients is $2-6 \%{ }^{7-9}$ Epidemiological surveys do not usually submit all uveitis patients to a strict protocol of tests to rigorously exclude sarcoidosis, and surveys where the diagnostic criteria are more stringent suggests that the true rate may be higher. ${ }^{10-12}$ Even after invasive studies, there remain a significant number of patients with uveitis and serological changes compatible with sarcoidosis but no clinical or histological confirmation of extraocular disease. ${ }^{11,13}$ Baarsma et al., ${ }^{13}$ in an unselected population of uveitis patients, found sarcoidosis in 5\% and a further $4 \%$ with an unexplained increase in sACE. Nordentoft and Møller ${ }^{10}$ found sarcoid granulomas in 14 of $43(33 \%)$ cases of idiopathic bilateral uveitis that underwent mediastinal lymph node biopsy. Weinreb and Kimura $^{12}$ found 12 of 27 (44\%) of patients with granulomatous uveitis had a raised SACE level but no evidence of systemic sarcoidosis. Ohara et al. ${ }^{11}$ performed transbronchial biopsy on 60 uveitis patients with no hilar lymphadenopathy and 'sparse supportive evidence' for sarcoidosis and found $62 \%$ had lung granulomas. Serum ACE levels were abnormal in $42 \%$ of those with a positive lung biopsy and also in a further $19 \%$ of those with a normal chest radiograph and negative biopsy. ${ }^{11}$ These studies suggest that the true prevalence of uveitis due to sarcoidosis is likely to be 2 or 3 times higher than that uncovered by pulmonary radiographic screening. In this study we employed wider inclusion criteria and could find no significant differences between the outcome of those patients with proven pulmonary disease and those with disease in other organs, or in those with less-defined disease. This confirms the finding of Stavrou et al. ${ }^{14}$ who found no difference in the clinical characteristics or outcome between those patients with a raised SACE and unsuspected extraocular disease (at onset) and those patients who had biopsy-proven extraocular sarcoidosis.

Uveitis and sarcoidosis may be short-lived when it accompanies the acute early symptoms of fever, rash and arthralgia, but most reports emphasise the frequency of chronic disease persisting for several years. Karma et al. ${ }^{2}$ found 8 of 21 had a monophasic disease but it was not clear whether the visual outcome in these cases was universally benign. Dana et al. ${ }^{15}$ found only $9 \%$ with acute disease and the mean time of active disease was 35 months. Our study suggests that with sufficient followup only a minority of patients have a benign ocular outcome in the long term. Although over half the patients retained normal acuity at their last follow-up, the survival analysis suggests that visual loss will occur in $70 \%$ of patients and over $80 \%$ will eventually require systemic steroids to control their disease. The frequency of oral steroids used in our study compares very closely with other reports; Karma found $66 \%$ needed steroids with a follow-up of 9 years, Ohara found that $51 \%$ needed oral steroids with a mean follow-up of 4 years, and Dana found $60 \%$ with a similar mean length of follow-up. ${ }^{2,11,15}$

The period during which patients may first need oral steroids appears to be at least 10 years. The period during which they may experience further visual loss or surgery appears to be as much as 18 years. This implies the need for the continued clinical supervision of these patients and emphasises how studies evaluating immunosuppressive and surgical therapy require extensive follow-up if they are to produce clinically relevant results.

Not all the outcomes have such a long time course. The requirement for an additional immunosuppressant to control disease does not appear to increase after 9 years, and there appears to be little risk of developing severe bilateral visual loss after the first 3 years following the onset of uveitis. This suggests that the most aggressive forms of uveitis only occur in the first few years, and posterior segment inflammation developing after this time is more likely to be controlled by steroids alone. The high rate of steroid treatment in sarcoid uveitis is similar to that found in neurosarcoidosis, where Chapelon et al. ${ }^{16}$ found that 31 of 35 (89\%) needed steroids and additional immunosuppression was required in 6 of $35(17 \%)$. The high rates of steroid usage in sarcoid uveitis and neurosarcoid contrast with those used in pulmonary sarcoidosis, where spontaneous resolution is much more frequent and only $15 \%$ need oral steroids. ${ }^{17}$

The visual prognosis in our study, and more recent studies, ${ }^{14,15}$ is a little better than found previously. Jabs found only $74 \%$ of patients had a visual acuity better than $6 / 36$, Karma found $73 \%$ of eyes better than $6 / 24$ and Rothova found $90 \%$ of patients better than $6 / 60{ }^{2,4,16}$ We found that $75 \%$ retained driving acuity (better than $6 / 12$ in one eye) for at least 12 years. The number with severe bilateral visual loss was less than $5 \%$ in our study and $5 \%$ and $6 \%$ in other recent studies. ${ }^{14,15}$ Dana et al. ${ }^{15}$ found that visual loss was strongly associated with delayed referral to a tertiary referral centre, but we could find no differences in outcome between the two centres in this study.

We confirm that visual loss is associated with surgery, raised intraocular pressure and posterior segment disease (inferred from the use of systemic steroids), but found few clear associations with the rate of visual loss apart from age and confirmed that the extent of extraocular disease bore little relation to the presence or severity of the uveitis. The lower rates of severe visual loss in recent studies may be due to the lower rates of glaucoma in these patient populations as much as changes in immunosuppressive treatment, since others have noted the close association between glaucoma and visual loss in this disease. ${ }^{1,4}$

We did not study the risk factors for developing uveitis in sarcoidosis but it has been found that nonwhite race, young age at onset, hypercalcaemia and chronic sarcoid elsewhere are associated with developing 
uveitis. ${ }^{3,18}$ We found a slight increase in females and in non-whites compared with the local population, but whites and males predominated in those with visual loss.

In our study the commonest new site for sarcoidosis was the central nervous system and there did not appear to be any diminution of risk over at least two decades. Previous studies have noted the association of posterior uveitis and neurosarcoidosis, ${ }^{19,20}$ but Stavrou et al. ${ }^{14}$ only found 1 of 40 patients developing neurosarcoid after the onset of uveitis. As neurological rather than pulmonary disease is the major site of new symptoms it would appear to be more appropriate to investigate for occult neurosarcoid rather than pulmonary sarcoid in those patients who are first assessed several years after the onset of uveitis. If patients do not present with pulmonary disease within 2 years of the onset of uveitis they are unlikely to develop it later on. Unfortunately neurosarcoidosis is notoriously difficult to diagnose and consequently the most likely form of the disease to remain undiagnosed pre-mortem. ${ }^{21} \mathrm{~A}$ high index of clinical suspicion must be maintained, especially in those patients with visual loss from uveitis with coincident optic neuropathy.

Patients were recruited into this study over a period of 3 years in two centres. There will, therefore, be an excess of patients with persistent disease and a corresponding reduction in those with acute, uncomplicated uveitis. The results compare well with the cohort study of Karma et al., ${ }^{2}$ suggesting that this recruitment bias is not great. Further cohort studies are required to investigate the course of sarcoid uveitis, but these will require multi-centre collaboration, as the estimated annual incidence of uveitis associated with confirmed extraocular sarcoidosis is 2 per million (unpublished data).

Sarcoid uveitis remains a serious disease and a difficult condition to diagnose definitively. To provide prognostic information for both patients and doctors, it would be justifiable to perform relatively non-invasive investigations such as conjunctival biopsy and serological testing. As clinical disease is most likely to be present in the lungs at presentation and has little chance of developing 2 or more years after the onset of uveitis, then the value of chest radiography - as a diagnostic test - when performed at a later date must be critically evaluated. It is not common practice to screen for asymptomatic pulmonary sarcoidosis in the normal population, nor to screen for neurosarcoidosis in those with known sarcoidosis. It may, therefore, be justifiable to restrict diagnostic testing in uveitis patients to repeated serological examination whilst retaining a high index of suspicion for new symptoms rather than subjecting all those at risk to lung biopsy or radionuclide scanning.

\section{References}

1. Rothova A, Suttorp-van Schulten MSA, Treffers WF, Kijlstra A. Causes and frequency of blindness in patients with intraocular inflammatory disease. $\mathrm{Br} \mathrm{J}$ Ophthalmol 1996;80:332-6.

2. Karma A, Huhti E, Poukkula A. Course and outcome of ocular sarcoidosis. Am J Ophthalmol 1988;106:467-72.

3. Kaye GB, Salmon JF, Isaacs S. Risk factors for uveitis in sarcoidosis. South Afr Med J 1993;83:342-4.

4. Jabs DA, Johns CJ. Ocular involvement in chronic sarcoidosis. Am J Ophthalmol 1986;102:297-301.

5. Iwai K, Tachibana T, Hosoda Y, Matsui Y. Sarcoidosis autopsies in Japan. Sarcoidosis 1988;5:60-5.

6. Hagerstrand I, Linell F. The prevalence of sarcoidosis autopsy material from a Swedish town. Acta Med Scand 1964;(Suppl 425):171-4.

7. Rothova A, Buitenhuis HJ, Meenken C, Brinkman CJJ, Linssen A, Alberts C, et al. Uveitis and systemic disease. Br J Ophthalmol 1992;76:137-41.

8. Tran VT, Auer C, Guex-Crosier Y, Pettet N, Herbort CP. Epidemiology of uveitis in Switzerland. Ocular Immunol Inflamm 1994;2:169-76.

9. Paivonsalo-Hietanen T, Vaahtoranta-Lehtonen $H$, Tuominen J, Saari KM. Uveitis survey at the University Eye Clinic in Turku. Acta Ophthalmol 1994;72:505-12.

10. Nordentoft V, Møller PM. Mediastinoscopy with lymph node biospy carried out in patients with endogenous uveitis of unknown aetiology. Acta Ophthalmol 1970;48:331-43.

11. Ohara K, Okubo A, Kamata K, Sasaki H, Kobayashi J, Kitamura S. Transbronchial lung biopsy in the diagnosis of suspected ocular sarcoidosis. Arch Ophthalmol 1993;111:642-4.

12. Weinreb RN, Kimura SJ. Uveitis associated with sarcoidosis and angiotensin converting enzymes. Am J Ophthalmol 1980;89:180-5.

13. Baarsma GS, La Hey E, Glasius E, de Vries J, Kijlstra A. The predictive value of serum angiotensin converting enzyme and lysozyme levels in the diagnosis of ocular sarcoidosis. Am J Ophthalmol 1987;104:211-7.

14. Stavrou P, Linton S, Young DW, Murray PI. Clinical diagnosis of ocular sarcoid. Eye 1997;11:365-70.

15. Dana M-R, Merayo-Lloves J, Schaumberg DA, Foster CS. Prognosticators for visual outcome in sarcoid uveitis. Ophthalmology 1996;103:1846-53.

16. Chapelon C, Ziza JM, Piette JC, Levy Y, Raguin G, Wechsler $B$, et al. Neurosarcoidosis: signs, course and treatment in 35 confirmed cases. Medicine 1990;69:261-76.

17. Hunninghake GW, Gilbert S, Pueringer R, Dayton C, Floerchinger C, Helmers R, et al. Outcome of the treatment for sarcoidosis. Am J Respir Crit Care Med 1994;149:893-8.

18. Rothova A, Alberts C, Glasius E, Kijlstra A, Buitenhuis HJ, Breebart AC. Risk factors for ocular sarcoidosis. Doc Ophthalmol 1989;72:287-96.

19. James DG, Friedman AI, Graham E. Uveitis: a series of 368 patients. Trans Ophthalmol Soc UK 1976;96:108-12.

20. James DG, Sharma OP. Neurosarcoidosis. Proc R Soc Med 1967;60:1169-70.

21. Manz HJ. Pathobiology of neurosarcoidosis and clinicopathological correlations. Can J Neurol Sci 1983;10:50-5. 